\title{
Drug poisoning deaths in the United States, 1999-2012: a statistical adjustment analysis
}

Christopher J. Ruhm ${ }^{1,2}$

\begin{abstract}
Background: Drug poisoning mortality in the US has risen rapidly but the drugs involved are frequently unspecified on death certificates.

Methods: Reported and adjusted proportions of specific drug types involved in fatal drug poisonings were calculated using vital statistics mortality data from 1999 to 2012. The adjusted proportions were those predicted to occur if at least one specific type of drug had been identified on the death certificates of all poisoning fatalities.

Results: Adjusted involvement rates of opioid analgesic mentions in 2012 were $54.3 \%$ (95\% confidence interval [CI]: $53.6 \%-55 \%$ ), $40.8 \%$ higher than the reported $38.6 \%$ rate. Adjusted rates for all narcotics, other narcotics, sedatives, or psychotropics, and multiple drug use were $81.5 \%$ (95 \% Cl: 80.9 \%-82.2 \%), $38.4 \%$ (95 \% Cl: $37.8 \%-39 \%$ ), $30 \%$ (95 \% Cl: $29.4 \%-30.7 \%$ ), 26 \% (95 \% Cl: $25.4 \%-26.6 \%$ ) and $42.8 \%$ (95 \% Cl: $42.1 \%-43.5 \%$ ) in 2012, compared to reported proportions of $60.7,27.9,18.7,18$ and $26.9 \%$. The adjustments typically had similar or slightly smaller effects on the estimates in 1999, and larger impacts on subcategories of drug types such benzodiazepines and antipsychotic medications. Based on the adjusted proportions, 22,534, 15,933, 12,457, 10,798, and 17,670 drug deaths in 2012 were estimated to involve opioid analgesics, other narcotics, sedatives, psychotropic medications, and drug combinations, compared to death certificate reports of 16,007, 11,567, 7,754, 7,467, and 11,176.

Conclusions: Death certificates substantially understate the involvement of opioid analgesics, sedatives, psychotropics, and drug combinations in fatal drug poisonings. Adjustment procedures that account for cases where only unspecified drugs are reported on death certificates provide more accurate information.
\end{abstract}

Keywords: Fatal drug poisonings, Drug poisoning mortality, Drug involvement, Poisoning deaths, Death certificates

\section{Background}

The poisoning mortality rate roughly tripled over the last three decades, with about $90 \%$ of these fatalities now caused by drugs [1]. At least $80 \%$ of drug fatalities were accidental in 2011 and these are now the leading cause of injury deaths [2]. Increased rates of poisoning deaths are the most important reason for the striking result that the all-cause mortality rates of $45-54$ year old non-Hispanic whites rose by around $0.5 \%$ per year between 1999 and 2013 [3]. The involvement of

\footnotetext{
Correspondence: ruhm@virginia.edu

${ }^{1}$ Frank Batten School of Leadership and Public Policy, University of Virginia, Garrett Hall 204, 235 McCormick Road, P.O. Box 400893, Charlottesville, VA 22904-4893, USA

${ }^{2}$ National Bureau of Economic Research, Cambridge, MA, USA
}

prescription opioid analgesics, such as oxycodone, methadone, and hydrocodone has received considerable attention [1, 4-7]. But drug poisonings are not limited to opioids - sedatives and psychotropic drugs are frequently identified on death certificates, and combination drug use is common $[8,9]$.

There are several barriers to formulating the most effective policies to deter dangerous use of prescription pharmaceuticals while avoiding the potential substitution to other harmful legal or illegal drugs. Perhaps most important is that we do not currently have reliable information on the specific drugs involved in fatal drug poisonings because no specific drug is identified on almost one-quarter of death certificates. As a result, rates of involvement for specific legal and illegal drugs 
are underestimated, as is the simultaneous use of drug combinations.

This study provides a more accurate understanding of the nature of drug poisoning deaths by using statistical methods that account for cases where no specific drug is identified on death certificates, to estimate the proportions of specific drug mentions and combination drug use for all types of drug poisoning mortality, as well as separately by manner of death (accidental, intentional or undetermined) and for two age groups (15-59 and $\geq 60$ year olds). Adjusted counts of fatal drug poisonings involving specified drug types are also calculated and contrasted with those based on death certificate reports. Data come from the 1999-2012 Centers for Disease Control and Prevention (CDC) Multiple Cause of Death $(M C O D)$ files, described in detail elsewhere [10].

\section{Methods}

The $M C O D$ files are based upon death certificates. Each certificate contains a single underlying cause of death, up to 20 additional causes, and demographic data. Information is utilized on cause of death, using four-digit International Classification of Diseases, Tenth Revision (ICD-10) codes, place of residence, age, race/ethnicity, gender, year, and weekday of death. The public use files lack geographic identifiers for most years; however, information on the state and county of residence, available under restricted conditions, was obtained and used for this analysis. The Institutional Review Board for the Social and Behavioral Sciences at the University of Virginia reviewed this project and determined that it does not involve the use of human subjects.

The investigation is limited to deaths occurring to US residents (foreign residents dying in the US were excluded). The main analysis begins in 1999 because ICD-9 codes, used prior to that year, are not fully comparable to ICD-10 categories [11]. Poisoning and drug poisoning deaths were defined using ICD-10 underlying cause of death (UCD) codes, where the UCD is the "disease or injury that initiated the chain of morbid events that led directly and inevitably to death" [12]. Poisoning deaths included ICD-10 codes X40-X49, X60-X69, X85X90 Y10-Y19; Y35.2; "U01(.6-.7); ICD-10 codes for drug poisoning deaths were X40-X44, X60-X64, X85, Y10-Y14; Y35.2; "U01(.6-.7) [13].

In cases of drug poisoning, the death certificate lists one or more drugs involved as immediate or contributory causes of death. These were included separately in the $M C O D$ files as ICD-10 "T-codes" and are referred to below as drug mentions. Specific drug categories examined include: narcotics, sedatives, psychotropics, other specified drugs, and unspecified drugs. Important subcategories of drug involvement were also analyzed. Narcotics were decomposed into (prescription) opioid analgesics and other narcotics; opioid analgesics into methadone and other opioid analgesics; other narcotics into heroin, cocaine, and other non-analgesic opioids. Benzodiazepines were sometimes separately broken out as an important subclass of sedatives. Among psychotropic medications, antidepressants, antipsychotics, and stimulants were separately examined. Other specified drugs comprised a wide variety of medications including anesthetics, antiallergic and immunosuppressive drugs, histamine and anti-gastric secretion medications, cardiac drugs, antibiotics and many others; deaths involving these drugs occurred relatively rarely and so were examined as a group. Poisoning by unspecified drugs, medicaments and biologicals (ICD-10 code, T50.9) was important because no specific drug was identified for approximately one-quarter of drug poisoning deaths. Combination drug use was examined through a constructed variable indicating mentions of two or more of the following drug categories: opioid analgesics, other opioids, sedatives, psychotropics, or other specified drugs.

Numbers and percentages of drug poisoning deaths were specified by type of drug, with the shares sometimes referred to as proportions below. Results are reported for 1999 and 2012, the first and last analysis years. Shares were also computed for subsamples of drug poisonings stratified by whether they were classified based on the underlying cause of death as accidental (UCD codes: X40-44), intentional (X60-64), of undetermined intent (Y10-Y14), or resulting from homicide/legal intervention (X85, Y35.2, "U01(.6-.7)). Proportions were also calculated separately for $16-59$ and $\geq 60$ year olds. Results are not shown for drug deaths involving persons younger than 16 because these occur too rarely (92 cases in 1999 and 147 in 2012) for adjusted proportions to be reliably estimated. Table 1 shows ICD-10 codes for each drug mention and manner of death category [14].

A dichotomous variable was constructed indicating if at least one specific drug was identified on the death certificate, rather than only the unspecified drug category being listed. State-year and county-year averages of this variable were calculated, with the latter denoted as SPECIFY. States were classified as "low diagnosis" if mention of a specific drug was provided in fewer than $68.8 \%$ of drug poisoning deaths in both 1999 and 2012 and as "high diagnosis" if this was done on more than $89.6 \%$ of cases in both years (Additional file 1). These thresholds reflected the $25^{\text {th }}$ and $75^{\text {th }}$ percentiles of drug specification rates over the 1999-2012 period. The within-state correlation in this variable across 1999 and 2012 was large but not perfect $(r=0.851)$.

Drug mention and manner of death shares were compared across high and low diagnosis states for 1999 and 2012 to provide a first indication of how reported proportions were affected by the frequent failure to 
Table 1 Drug involvement in drug poisoning deaths: 1999 and $2012^{\text {a }}$

\begin{tabular}{|c|c|c|c|c|}
\hline \multirow[t]{2}{*}{ Drug category/Manner of death } & \multicolumn{2}{|l|}{1999} & \multicolumn{2}{|l|}{2012} \\
\hline & Percent & Number & Percent & Number \\
\hline All drug deaths $(X 40-44, X 60-64, X 85, Y 10-14, Y 35.2, * \cup 01(.6-.7))^{b}$ & 100.0 & 16,849 & 100.0 & 41,502 \\
\hline \multicolumn{5}{|l|}{ Drug mentions (T-codes) } \\
\hline Narcotics (40.0-40.9) & 58.9 & 9922 & 60.7 & 25,187 \\
\hline Opioid analgesics (40.2-40.4) & 23.9 & 4030 & 38.6 & 16,007 \\
\hline Methadone (40.3) & 4.7 & 784 & 9.5 & 3932 \\
\hline Other opioid analgesics $(40.2,40.4)$ & 19.9 & 3360 & 31.4 & 13,012 \\
\hline Other narcotics (40.0-40.1, 40.5-40.9) & 42.4 & 7137 & 27.9 & 11,567 \\
\hline Heroin (40.1) & 11.6 & 1960 & 14.3 & 5925 \\
\hline Cocaine (40.5) & 22.7 & 3822 & 10.6 & 4404 \\
\hline Miscellaneous $(40.0,40.6-40.9)$ & 17.4 & 2931 & 7.2 & 3006 \\
\hline Sedatives (40.2-40.8) & 9.9 & 1662 & 18.7 & 7754 \\
\hline Benzodiazepines (42.4) & 6.7 & 1135 & 15.7 & 6524 \\
\hline Other sedatives (42.0-42.3, 42.5-42.8) & 3.9 & 663 & 4.9 & 2048 \\
\hline Psychotropics (43.0-43.9) & 14.6 & 2466 & 18.0 & 7467 \\
\hline Antidepressants (43.0-43.2) & 10.4 & 1749 & 10.3 & 4259 \\
\hline Antipsychotics (43.3-43.5) & 1.9 & 321 & 3.2 & 1333 \\
\hline Stimulants (43.6) & 3.2 & 547 & 6.3 & 2635 \\
\hline Other specified (36.0-38.9, 41.0, 41.9, 44.0-48.7, 49.0-50.8) & 6.9 & 1171 & 7.6 & 3156 \\
\hline Unspecified (50.9) & 50.3 & 8477 & 49.7 & 20,612 \\
\hline$>1$ Major drug class ${ }^{c}$ & 18.0 & 3040 & 26.9 & 11,176 \\
\hline \multicolumn{5}{|l|}{ Manner of death } \\
\hline Accidental (X40-44) & 66.2 & 11,155 & 79.9 & 33,175 \\
\hline Intentional (X60-64) & 18.9 & 3181 & 13.2 & 5465 \\
\hline Undetermined intent (Y10-Y14) & 14.7 & 2473 & 6.7 & 2782 \\
\hline Homicide (X85, Y35.2, *U01(.6-.7)) & 0.2 & 40 & 0.2 & 80 \\
\hline
\end{tabular}

${ }^{a}$ Data from the Multiple Cause of Death files

${ }^{b}$ Entries in parentheses refer to ICD-10 X and $\mathrm{Y}$ codes for the underlying causes of death used to classify drug poisoning and manner of death, and $\mathrm{T}$ codes for drug mentions

'Two or more of the drug types: opioid analgesics, other narcotics, sedatives, psychotropics, or other specified drugs

identify any of the specific drugs involved in fatal drug poisonings. Such comparisons will not be fully informative if high and low diagnosis states differ along other dimensions. To control for potential confounding factors, a series of probit models were separately estimated for 1999 and 2012.

Probit is a nonlinear regression model specifically designed for binary dependent variables, such as drug mentions. It is similar to logit (logistic) regression except that the cumulative distribution function of the error term has a standard normal, rather than logistic, distribution [15]. The basic probit model took the form:

$$
Y_{i j t}=\alpha+\beta \operatorname{SPECIFY} Y_{j t}+\gamma X_{i j t}+\mu_{i j t},
$$

where $Y_{i j t}$ was a binary dependent variable indicating if the death for individual $i$ in county $j$ and year $t$ was reported to involve the specified drug type. SPECIFY, the explanatory variable of primary interest, measured the county-year drug specification rate. $\mu$ is a regression error term.

Predictive power of the model was improved by including supplementary covariates $(X)$ capturing the effects of characteristics related to the likelihood of the specified drug being involved in the death but not caused by county differences in SPECIFY. The main specifications included controls for: gender, two race indicators (black and other nonwhite), being married at the time of death (versus never married, separated/divorced, widowed, or status not reported), four educational categories (less than high school graduate, high school graduate, some college, college graduate, with education not reported as the reference group), eight age categories $(\leq 20,21-30,31-40$, $41-50,51-60,61-70,71-80$, >80, with missing age as the 
reference group), nine census regions (New England, Mid-Atlantic, East North Central, West North Central, South Atlantic, East South Central, West South Central, Mountain and Pacific), and seven day of the week of death indicators. Education was sometimes reported in years rather than specific thresholds. In these cases, $\leq 11,12$, $13-15$, and $\geq 16$ years were classified as less than high school graduate, high school graduate, some college, and college graduate. Manner of death was not controlled for in the primary specifications, because of evidence provided below that classification varies with county-level drug specification rates and previous research indicating that death certificates sometimes misclassify intentional drug fatalities as being accidental or of undetermined intent $[16,17]$. Supplementary models added controls for the manner of death (accidental or intentional, with undetermined intent/homicide as the reference group) allowing for proportions to be compared with and without the inclusion of these supplementary controls.

Predicted values of the dependent variable were calculated for each drug poisoning death and then averaged over all observations to obtain estimated proportions.

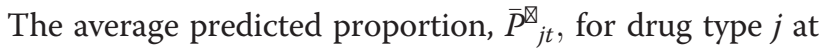
time $t$, was:

$$
\begin{aligned}
\bar{P}_{j t}^{\rrbracket_{j t}} & =\frac{1}{n} \sum_{i=1}^{n} \Phi\left(\hat{Y}_{i j t}\right) \\
& =\frac{1}{n} \sum_{i=1}^{n} \Phi\left(\hat{\alpha}+\hat{\beta} \text { Specif }_{j t}+\hat{\gamma} X_{i j t}\right),
\end{aligned}
$$

for $\Phi($.$) , the cumulative distribution function of the stand-$ ard normal distribution. Since these predictions were based on actual values of the explanatory variables, the estimated proportions were expected to be similar to the sample mean values. This was tested for and confirmed for all types of drug mentions in both 1999 and 2012 (Additional file 2).

Next, a second set of predicted values was obtained after setting SPECIFY to one for all observations. The average expected value, hereafter referred to as the "adjusted proportion", $\tilde{P}_{j t}$, was estimated as:

$$
\tilde{P}_{j t}=\frac{1}{n} \sum_{i=1}^{n} \Phi\left(\hat{\alpha}+\hat{\beta}+\hat{\gamma} X_{i j t}\right),
$$

and indicates the drug involvement rate expected if at least one specific drug type had been identified on all drug poisoning death certificates. Ninety-five percent confidence intervals (95\% CI) were calculated using the delta method.

The predicted number of deaths involving the specified class of drugs, $\tilde{D}_{j t}$, was computed as the product of the adjusted proportion and number of drug poisoning deaths in year $t, D_{t}$ or:

$$
\tilde{D}_{j t}=\tilde{P}_{j t} \times D_{t}
$$

The associated lower (upper) threshold of the $95 \%$ CI was computed as the product of the number of drug fatalities times the lower (upper) value of the adjusted proportion $95 \% \mathrm{CI}$.

Although there is no single agreed-upon measure of goodness-of-fit in probit or logit models (corresponding to $R^{2}$ in a regression framework), a common measure used here is the pseudo- $R^{2}$, defined as $1-\mathcal{L}_{u r} / \mathcal{L}_{0}$, where $\mathcal{L}_{u r}$ is the log-likelihood function of the estimated specification and $\mathcal{L}_{0}$ is the log-likelihood function of the intercept-only model [18]. Two additional indications of the success of the adjustment procedures were examined. The first compared reported and adjusted shares of exclusive unspecified drug mentions (those where no drug was identified on the death certificate). As mentioned, the reported proportion was approximately $25 \%$ in most years. Completely successful adjustment procedures would reduce this to zero, and estimated shares close to this would provide confidence in the adjustment procedure. The second test was the reverse of the first. Here, adjusted proportions were calculated using the same procedure but assuming that drug types were never specified on the death certificates, by predicting proportions after setting SPECIFY to zero as: $\tilde{\tilde{P}}_{j t}=\frac{1}{n} \sum_{i=1}^{n} \Phi\left(\hat{\alpha}+\hat{\gamma} X_{i j t}\right)$. Perfect adjustment would imply exclusive mentions of unspecified drugs in $100 \%$ of drug fatalities. Predicted probabilities can never reach zero or one in a probit model, so that complete adjustment will never be achieved.

Analyses were conducted using STATA Statistical Software: Release 14 [19]. Statistical significance refers to $P<.05$, from two-sided tests.

\section{Results}

Trends in drug poisoning and specific drug mention rates Drug deaths rose $146 \%$ between 1999 and 2012, from 16,849 to 41,502 . The national population grew by $12.5 \%$ and the total number of deaths by $6.4 \%$ over the same period, so that the (non-age-adjusted) fatal poisoning rate increased by $119 \%$ from 6.04 to 13.22 per 100,000 and from $0.70 \%$ to $1.55 \%$ of all deaths. Poisoning mortality of all types grew $134 \%$ (from 19,741 to 46,150) between 1999 and 2012, and drug fatalities as a share of poisoning deaths increased from 85 \% in 1999 to $92 \%$ in 2006, before declining slightly to $90 \%$ in 2012 (Fig. 1). The rise in drug mortality represents an acceleration of a trend that started in the early 1980s. In 1982, 6518 individuals died from drugs, or 2.81 per 100,000 population, and these constituted $58 \%$ of all poisoning fatalities [1]. The drug poisoning death rate grew $4.6 \%$ annually between 1982 and 1999 (from 2.81 to 6.04 per 100,000) versus $6.2 \%$ per year from 1999 to 2012 (from 6.04 to 13.22 per 100,000). 


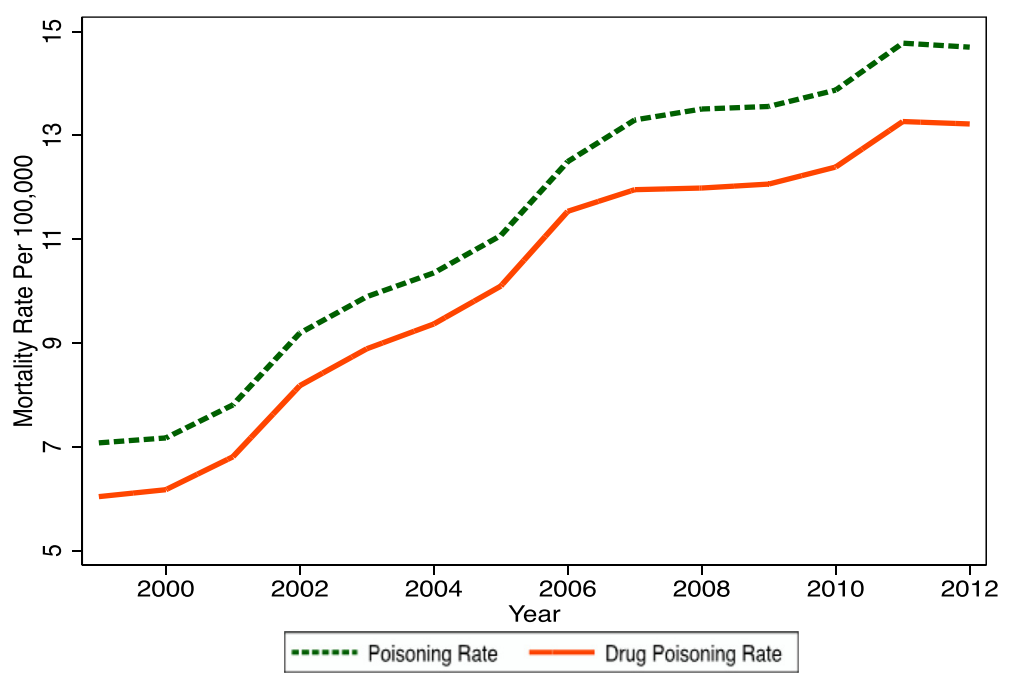

Fig. 1 Poisoning and drug poisoning mortality rates

One or more specific drugs were mentioned for 78.1, 73.1, and $75.8 \%$ of drug fatalities in 1999, 2008, and 2012 (Fig. 2).

\section{Death certificate reports}

Narcotics were identified in $60.7 \%$ of all 2012 drug fatalities, slightly above $58.9 \%$ in 1999 (Table 1). This reflected a rapid increase in opioid analgesic mentions (from $23.9 \%$ to $38.6 \%$ ) and heroin-involved deaths (from $11.6 \%$ to $14.2 \%)$ that more than offset substantial reductions in reported shares of cocaine and miscellaneous narcotics. However, since drug deaths increased dramatically between 1999 and 2012 (from 16,849 to 41,502), the number of drug mentions sometimes increased even while proportions declined. For example, cocaine mentions grew from
3822 to 4404 fatalities while the corresponding share of drug deaths fell from $22.7 \%$ to $10.6 \%$.

These overall patterns conceal heterogeneity within drug classes. Methadone mentions more than quintupled over the 13 years, while other opioid analgesic involvement less than quadrupled. The $367 \%$ increase in sedative involvement was largely due to the $475 \%$ rise in benzodiazepine mentions, and reported psychotropic drug mentions grew $203 \%$, led by $382 \%$ and $315 \%$ increases in stimulant and antipsychotic involvement. Mentions of other specified drugs rose $170 \%$. Also noteworthy was the rise from 8477 to 20,612 in unspecified drug involvement that exceeded opioid analgesic mentions by $29 \%$ in 2012 . Drug combinations were reported in $18 \%$ fatal drug poisonings in 1999 and $27 \%$ in 2012.

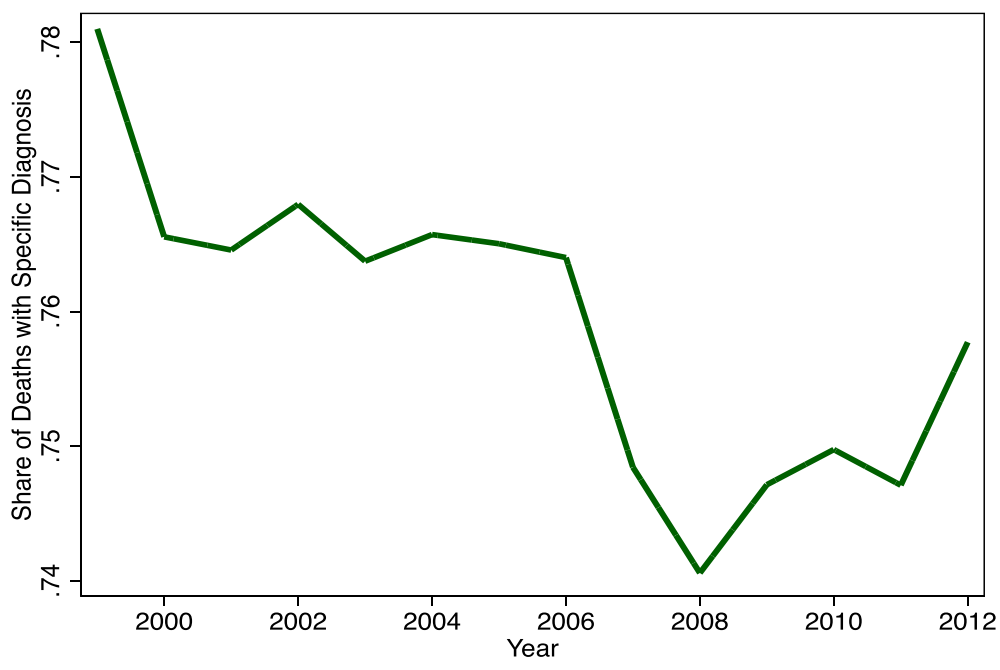

Fig. 2 Drug specification rate 
The classified manner of drug fatalities changed over time, with the share of accidental poisonings rising from $66 \%$ to $80 \%$ between 1999 and 2012, while intentional deaths fell from $19 \%$ to $13 \%$ and those of undetermined intent from $15 \%$ to $7 \%$; homicides represented a miniscule $0.2 \%$ of drug poisoning fatalities in both years (bottom panel of Table 1).

\section{Adjusted shares}

Death certificates understate most drug involvement proportions because of the frequency of cases where no specific drug was identified. A first indication of this was obtained by comparing death certificate reports in high and low diagnosis states (Table 2). As expected, the reported proportions were almost always greater in the high diagnosis locations. For example, narcotics involvement in 2012 was $78.3 \%$ in high diagnosis states versus $41.1 \%$ in those with low diagnosis rates. Substantial differences were obtained for opioid analgesics, sedatives, psychotropics, and combination drug use, while unspecified drugs were less commonly mentioned, with $36.5 \%$ for high versus $68.4 \%$ for low diagnosis in 2012. One exception is that heroin mentions were more prevalent in low than high diagnosis states in 1999 (but not 2012).

This comparison does not account for potential confounders. As evidence that these may matter, drug poisoning rates almost tripled in the low diagnosis states (from 5.0 to 14.1 per 100,000) between 1999 and 2012 compared to less than doubling (from 7.0 to 13.4 per $100,000)$ in high diagnosis areas. There are also substantial differences in the classified manner of deaths, with accidental fatalities being more common and those with undetermined intent being less frequent in low than in high diagnosis states. To the extent that these represent differences in reporting patterns rather than actual mechanisms of death, errors may be introduced when

Table 2 Drug involvement in drug poisoning deaths in high and low diagnosis states ${ }^{\mathrm{a}}$

\begin{tabular}{|c|c|c|c|c|}
\hline \multirow[t]{2}{*}{ Drug category/Manner of death } & \multicolumn{2}{|l|}{1999} & \multicolumn{2}{|l|}{2012} \\
\hline & Low diagnosis states ${ }^{\mathrm{b}}$ & High diagnosis states $^{c}$ & Low diagnosis states ${ }^{\mathrm{b}}$ & High diagnosis states ${ }^{c}$ \\
\hline \multicolumn{5}{|l|}{ Drug mentions } \\
\hline Narcotics & 35.9 & 76.1 & 41.1 & 78.3 \\
\hline Opioid analgesics & 13.4 & 23.8 & 24.7 & 52.1 \\
\hline Other narcotics & 25.0 & 60.7 & 20.0 & 36.5 \\
\hline Heroin & 10.8 & 7.9 & 12.2 & 18.5 \\
\hline Cocaine & 13.5 & 29.3 & 6.6 & 13.5 \\
\hline Sedatives & 5.6 & 11.0 & 10.6 & 24.8 \\
\hline Benzodiazepines & 3.8 & 7.4 & 9.0 & 20.2 \\
\hline Psychotropics & 7.7 & 17.0 & 8.5 & 23.9 \\
\hline Antidepressants & 6.1 & 13.2 & 5.9 & 14.7 \\
\hline Antipsychotics & 1.0 & 2.4 & 1.5 & 4.6 \\
\hline Stimulants & 1.0 & 3.2 & 1.9 & 7.2 \\
\hline Other specified & 7.4 & 6.4 & 4.9 & 11.0 \\
\hline Unspecified & 66.7 & 30.7 & 68.4 & 36.5 \\
\hline$>1$ Major drug class ${ }^{d}$ & 8.1 & 21.5 & 15.1 & 39.8 \\
\hline Drug poisoning rate & 5.04 & 7.00 & 14.09 & 13.37 \\
\hline \multicolumn{5}{|l|}{ Manner of death } \\
\hline Accidental & $64.1 \%$ & $49.6 \%$ & $80.6 \%$ & $73.5 \%$ \\
\hline Intentional & $19.1 \%$ & $16.1 \%$ & $10.9 \%$ & $13.5 \%$ \\
\hline Undetermined intent & $16.2 \%$ & $34.0 \%$ & $8.3 \%$ & $12.9 \%$ \\
\hline Homicide & $0.6 \%$ & $0.3 \%$ & $0.2 \%$ & $0.1 \%$ \\
\hline
\end{tabular}

Data from the Multiple Cause of Death files

bow diagnosis states, defined as those with at least one drug specified for fewer than $68.8 \%$ of drug poisoning deaths in both 1999 and 2012 , include: Alabama, Idaho, Indiana, Louisiana, Michigan, Mississippi, Montana, Nebraska, North Dakota and Pennsylvania

'High diagnosis states, defined as those with at least one drug specified for more than $89.6 \%$ of drug poisoning deaths in both 1999 and 2012 include: Alaska, Maryland, Massachusetts, Nevada, New Hampshire, New York, Oklahoma, Rhode Island, Utah, Virginia and Washington

${ }^{\mathrm{d}}$ Two or more of the drug types: opioid analgesics, other narcotics, sedatives, psychotropics, or other specified drugs

e Drug poisoning rate is per 100,000 population 
including the controls for the manner of death when estimating adjusted proportions or when examining differences in shares across reported manners of drug deaths.

Table 3 displays reported and adjusted proportions, as well as percentage differences between the two, for 1999 and 2012. As mentioned, adjusted involvement was estimated from probit models that account for spurious correlation related to differences in age, sex, race/ethnicity, educational attainment, census region, and the weekday of the death, and calculated under the assumption that at least one specific drug was identified for each drug poisoning fatality.

The adjustment procedures significantly raised the predicted frequency of all specific drug proportions, implying that death certificates understated most types of drug involvement. For example, the adjusted proportion of opioid analgesic mentions in 2012 was $54.3 \%$ (95\% CI, $53.6 \%-55 \%$ ), or $40.8 \%$ (95\% CI, $39 \%-42.5 \%$ ) higher than the reported $38.6 \%$. (Percentage differences vary slightly on the table due to rounding. In the example just shown, adjusted and reported proportions were actually $54.296 \%$ and $38.569 \%$, and the difference between them was $40.776 \%)$.

Adjusted proportions for narcotics, psychotropics, other specified drugs, and combination drug use exceeded reported shares by $34 \%$ to $46 \%$, while those for unspecified drugs fell $31 \%$. The increase was a particularly large, at $60.7 \%$ (95\% CI, $57.1 \%-64.2 \%$ ) for sedatives - mostly due to the $62.6 \%$ (95 \% CI, $58.6 \%-66.5 \%$ ) growth for benzodiazepines - and also for antipsychotics and antidepressants, where adjusted proportions exceeded reported values by $65.1 \%$ (95\% CI, $54.7 \%-75.5 \%$ ) and $60.2 \%$ (95\% CI, $55 \%-65.4 \%$ ). The adjustment procedure typically had similar or slightly smaller percentage effects on drug shares in 1999.

An alternative set of adjusted proportions was obtained from models that contained additional controls for the manner of death (accidental or intentional, with undetermined intent/homicides as the reference group). This specification has the potential advantage of increased predictive power, but will be problematic if classification of the manner of death depends on drug specification rates, as was suggested above. In practice, the adjusted shares were virtually identical to those obtained from the main model (Additional file 3).

Table 4 details adjusted proportions and percentage differences compared to death certificate reports in 1999 and 2012 for subsamples stratified by whether the fatal drug poisoning was classified as accidental, intentional, or of undetermined intent. Results for homicides are not reported since these accounted for just $0.2 \%$ of drug mortality. (Additional file 4 shows reported proportions). Patterns of drug involvement vary substantially

Table 3 Reported and adjusted drug involvement ${ }^{\mathrm{a}}$

\begin{tabular}{|c|c|c|c|c|c|c|}
\hline \multirow[t]{3}{*}{ Drug category } & \multicolumn{6}{|l|}{$\%[95 \% \mathrm{Cl}]$} \\
\hline & \multicolumn{3}{|l|}{1999} & \multicolumn{3}{|l|}{2012} \\
\hline & Reported $^{\mathrm{b}}$ & Adjusted $^{c}$ & $\%$ Difference $^{d}$ & Reported $^{\mathrm{b}}$ & Adjusted $^{c}$ & $\%$ Difference $^{d}$ \\
\hline Narcotics & 58.9 & 77.8 [76.9-78.7] & $32.2[30.6-33.7]$ & 60.7 & 81.5 [80.9-82.2] & $34.3[33.2-35.4]$ \\
\hline Opioid analgesics & 23.9 & $31.3[30.2-32.4]$ & 30.9 [26.2-35.6] & 38.6 & $54.3[53.6-55.0]$ & $40.8[39.0-42.5]$ \\
\hline Other narcotics & 42.4 & $58.3[57.2-59.3]$ & $37.6[35.1-40.1]$ & 27.9 & 38.4 [37.8-39.0] & $37.7[35.5-40.0]$ \\
\hline Heroin & 11.6 & $14.1[13.3-14.9]$ & $20.9[14.2-27.7]$ & 14.3 & $20.0[19.4-20.5]$ & 39.9 [36.2-43.6] \\
\hline Cocaine & 22.7 & $31.1[30.0-32.1]$ & $37.0[32.4-41.6]$ & 10.6 & $14.9[14.4-15.3]$ & $40.0[35.5-44.5]$ \\
\hline Sedatives & 9.9 & 15.6 [14.7-16.6] & 58.4 [48.8-68.0] & 18.7 & 30.0 [29.4-30.7] & $60.7[57.1-64.2]$ \\
\hline Benzodiazepines & 6.7 & $11.4[10.6-12.3]$ & $69.6[56.8-82.3]$ & 15.7 & 25.6 [24.9-26.2] & $62.6[58.6-66.5]$ \\
\hline Psychotropics & 14.6 & 21.2 [20.1-22.2] & $44.7[37.7-51.7]$ & 18.0 & 26.0 [25.4-26.6] & $44.6[41.2-48.0]$ \\
\hline Antidepressants & 10.4 & $16.3[15.4-17.3]$ & $57.1[47.9-66.3]$ & 10.3 & $16.4[15.9-17.0]$ & $60.2[55.0-65.4]$ \\
\hline Antipsychotics & 1.9 & $3.2[2.7-3.7]$ & $68.9[43.0-94.7]$ & 3.2 & $5.3[5.0-5.6]$ & $65.1[54.7-75.5]$ \\
\hline Stimulants & 3.2 & $3.9[3.4-4.4]$ & $19.9[5.3-34.6]$ & 6.3 & $7.9[7.5-8.3]$ & $24.4[18.6-30.2]$ \\
\hline Other specified & 6.9 & 8.8 [8.1-9.5] & 26.6 [16.8-36.3] & 7.6 & 10.9 [10.5-11.3] & 43.5 [37.8-49.2] \\
\hline Unspecified & 50.3 & 35.4 [34.3-36.5] & $-29.6[-31.7--27.4]$ & 49.7 & 34.1 [33.4-34.7] & $-31.4[-32.7--30.2]$ \\
\hline$>1$ Major drug class ${ }^{\mathrm{e}}$ & 18.0 & 28.6 [27.5-29.8] & 58.6 [52.2-64.9] & 26.9 & $42.8[42.1-43.5]$ & $58.9[56.3-61.5]$ \\
\hline
\end{tabular}

${ }^{\mathrm{a}}$ Data from the Multiple Cause of Death files

${ }^{\mathrm{b}}$ Proportions from death certificate reports

${ }^{C}$ Adjusted proportions are average predicted values from probit models, where at least one specific drug is assumed to be mentioned for all poisoning deaths (SPECIFY = 1). Models also control for: sex, race (black, other), Hispanic, currently married, education (high school dropout, high school graduate, some college, college graduate), age $(\leq 20,21-30,31-40,41-50,51-60,61-70,71-80,>80)$, day of the week of death, and census region

$\mathrm{d}_{\%}$ Difference between adjusted and reported proportions (calculated using more precise proportions than the rounded percentages displayed on the table)

' $T$ wo or more of the drug types: opioid analgesics, other narcotics, sedatives, psychotropics, or other specified drugs 
Table 4 Adjusted drug involvement by manner of death and change vs. reported proportion ${ }^{a}$

\begin{tabular}{|c|c|c|c|c|c|c|}
\hline \multirow[t]{3}{*}{ Drug category } & \multicolumn{6}{|l|}{$\%[95 \% \mathrm{Cl}]$} \\
\hline & \multicolumn{2}{|l|}{ Accidental } & \multicolumn{2}{|l|}{ Intentional } & \multicolumn{2}{|c|}{ Undetermined intent } \\
\hline & Adjusted $^{b}$ & $\%$ Difference ${ }^{c}$ & Adjusted $^{b}$ & $\%$ Difference ${ }^{c}$ & Adjusted $^{b}$ & $\%$ Difference ${ }^{c}$ \\
\hline \multicolumn{7}{|l|}{1999} \\
\hline Narcotics & 86.9 [86.0-87.7] & 29.9 [28.6-31.2] & $36.2[33.3-39.2]$ & $52.2[40.1-64.9]$ & $81.0[78.6-83.4]$ & 18.8 [15.4-22.3] \\
\hline Opioid analg. & $34.6[33.2-36.0]$ & $33.1[27.7-38.5]$ & $28.9[26.1-31.7]$ & $52.1[37.2-66.9]$ & $25.7[23.0-28.4]$ & $23.1[10.2-35.9]$ \\
\hline Other narcotics & 68.6 [67.4-69.9] & 36.0 [33.5-38.4] & $9.8[7.9-11.6]$ & 62.9 [32.0-93.7] & $63.1[60.3-65.8]$ & 19.8 [14.6-25.0] \\
\hline Sedatives & 13.3 [12.2-14.4] & 67.6 [53.6-81.7] & 30.8 [28.0-33.7] & $62.0[47.0-77.1]$ & 10.7 [8.5-12.9] & $56.8[24.5-89.2]$ \\
\hline Psychotropics & $15.9[14.7-17.0]$ & $46.7[36.2-57.2]$ & $45.0[42.0-48.0]$ & $55.7[45.3-66.1]$ & 18.8 [16.2-21.3] & $40.2[21.0-59.4]$ \\
\hline Other specified & $6.9[6.2-7.6]$ & 17.5 [5.8-29.1] & $18.7[16.3-21.1]$ & $45.4[26.9-64.0]$ & $5.9[4.3-7.6]$ & $48.2[7.2-89.1]$ \\
\hline Unspecified & $33.1[31.8-34.4]$ & $-32.6[-29.9--35.2]$ & $55.0[52.1-57.9]$ & $-16.4[-12.0--20.9]$ & $25.0[22.4-27.5]$ & $-31.3[-24.3--38.4]$ \\
\hline$>1$ Drug class $^{\mathrm{d}}$ & $30.1[28.7-31.5]$ & $57.8[50.4-65.3]$ & $33.6[30.5-36.7]$ & $92.9[75.2-110.6]$ & $22.6[19.8-25.4]$ & $59.5[39.8-79.2]$ \\
\hline \multicolumn{7}{|l|}{2012} \\
\hline Narcotics & 87.4 [86.9-87.8] & 32.9 [32.2-33.6] & $44.4[42.5-46.3]$ & 38.7 [32.8-44.6] & 76.9 [74.7-79.1] & 35.8 [32.0-39.6] \\
\hline Opioid analg. & $56.5[55.8-57.3]$ & $41.4[39.6-43.3]$ & $40.0[38.1-41.9]$ & $39.6[33.1-46.2]$ & $57.7[55.1-60.3]$ & $38.5[32.3-44.8]$ \\
\hline Other narcotics & $43.9[43.2-44.6]$ & 36.3 [34.0-38.5] & $6.2[5.2-7.1]$ & 40.3 [18.7-62.0] & 30.8 [28.4-33.1] & $39.0[28.3-49.6]$ \\
\hline Sedatives & 29.7 [29.0-30.4] & 64.8 [60.7-68.9] & $36.2[34.3-38.0]$ & $48.6[40.9-56.2]$ & $24.6[22.2-27.0]$ & 56.4 [41.1-71.7] \\
\hline Psychotropics & 23.6 [23.0-24.3] & 44.8 [40.8-48.9] & 41.4 [39.5-43.3] & $46.9[40.2-53.6]$ & $27.0[24.6-29.5]$ & 50.9 [37.3-64.6] \\
\hline Other specified & $8.2[7.8-8.7]$ & $51.8[43.9-59.8]$ & $28.8[27.1-30.5]$ & $33.4[25.5-41.3]$ & $8.9[7.3-10.5]$ & $46.6[20.2-73.0]$ \\
\hline Unspecified & $32.2[31.5-32.9]$ & $-33.3[-31.9--34.8]$ & $48.6[46.7-50.4]$ & $-18.2[-15.1--21.3]$ & $30.6[28.2-32.9]$ & $-35.4[-30.5--40.4]$ \\
\hline$>1$ Drug class $^{d}$ & $43.2[42.4-44.0]$ & $60.2[57.3-63.1]$ & $43.3[41.3-45.2]$ & 56.6 [49.7-63.6] & $39.2[36.5-41.9]$ & 53.2 [42.8-63.7] \\
\hline
\end{tabular}

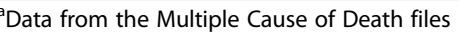

${ }^{b}$ Adjusted proportions are average predicted values from probit models, where at least one specific drug is assumed to be mentioned for all poisoning deaths (SPECIFY = 1). Models also control for: sex, race (black, other), Hispanic, currently married, education (high school dropout, high school graduate, some college, college graduate), age $(\leq 20,21-30,31-40,41-50,51-60,61-70,71-80,>80)$, day of the week of death, and census region

$c_{\%}$ Difference shows the percentage difference between adjusted proportions and those obtained directly from mentions on death certificates

${ }^{\mathrm{d}}$ Two or more of the drug types: opioid analgesics, other narcotics, sedatives, psychotropics, or other specified drugs

by manner of death, with narcotics - particularly nonanalgesics - being much more commonly involved for accidental fatalities and those of undetermined intent; whereas sedatives, psychotropics, and other specified and unspecified drugs played bigger roles for intentional drug deaths. However, there were no clear patterns of percentage differences in adjusted versus reported proportions across years or manners of death.

Adjusted shares and percentage differences versus reported drug involvement are shown separately for 16-59 and $\geq 60$ year olds in Table 5 . (Additional file 5 provides corresponding reported proportions). Information for persons younger than 16 is not displayed since they accounted for less than $0.6 \%$ of drug fatalities. There were more than eight times as many drug deaths among $16-59$ year olds (15,484 in 1999 and 37,171 in 2012) as $\geq 60$ year olds (1257 in 1999 and 4182 in 2012), generally resulting in more precise adjusted proportions for the younger group.

Compared to drug deaths involving persons 60 and over, those among 16-59 year olds more often involved narcotics of all kinds and combination drug use, with other specified drugs less often implicated but with no clear patterns for sedatives, psychotropics or unspecified drugs. Reported proportions understate drug involvement by similar amounts for the two age groups with two exceptions: there was a particularly large downwards bias in the share of other drugs for 16-59 year olds in 1999 and 2012, as well as for sedatives in 1999.

\section{Number of deaths}

The first and third columns of Table 6 show estimates for the number of drug poisoning deaths involving major categories of drugs, based on the adjusted proportions detailed in Table 3. The second and fourth columns indicate how these differ from the corresponding numbers reported on death certificates. To illustrate, 22,534 drug deaths were estimated to involve opioid analgesics in 2012, calculated as the adjusted proportion (54.296 \%) times the total number of drug poisoning deaths $(41,502)$. This number is 6527 above the 16,007 death certificates mentioning this type of drug. The calculations use more significant digits than reported on the tables, so that the small differences occur compared to calculations based on the rounded estimates. 
Table 5 Adjusted drug involvement by age group and change vs. reported proportion ${ }^{a}$

\begin{tabular}{|c|c|c|c|c|}
\hline \multirow[t]{3}{*}{ Drug category } & \multicolumn{4}{|l|}{$\%$ [95 \% ci] } \\
\hline & \multicolumn{2}{|l|}{ 16-59 year olds } & \multicolumn{2}{|l|}{$\geq 60$ year olds } \\
\hline & Adjusted $^{b}$ & $\%$ Difference ${ }^{c}$ & Adjusted $^{b}$ & $\%$ Difference $^{c}$ \\
\hline \multicolumn{5}{|l|}{1999} \\
\hline Narcotics & 81.1 [80.2-82.0] & 31.4 [29.9-32.8] & 36.5 [32.3-40.7] & $39.5[23.3-55.7]$ \\
\hline Opioid analgesics & $32.0[30.8-33.2]$ & $30.4[25.6-35.1]$ & 23.4 [19.5-27.3] & 42.0 [18.1-65.9] \\
\hline Other narcotics & $61.8[60.7-62.9]$ & 37.3 [34.8-39.8] & 16.6 [13.6-19.6] & 40.7 [15.2-66.2] \\
\hline Sedatives & $15.2[14.3-16.2]$ & $60.8[50.5-71.2]$ & 20.8 [17.0-24.6] & 39.2 [13.8-64.6] \\
\hline Psychotropics & $21.3[20.3-22.4]$ & $44.4[37.2-51.7]$ & $20.3[16.5-24.1]$ & $52.8[24.1-81.5]$ \\
\hline Other specified & $6.7[6.1-7.4]$ & 38.3 [24.8-51.9] & $32.3[28.2-36.4]$ & $3.8[-9.2-16.9]$ \\
\hline Unspecified & $35.4[34.3-36.6]$ & $-30.5[-28.2--32.7]$ & 36.7 [32.5-40.8] & $-15.9[-6.4--25.3]$ \\
\hline$>1$ Major drug class ${ }^{d}$ & $29.6[28.4-30.8]$ & $58.2[51.8-64.7]$ & $18.3[14.6-22.1]$ & $63.5[30.0-97.0]$ \\
\hline \multicolumn{5}{|l|}{2012} \\
\hline Narcotics & 83.4 [82.9-84.0] & $34.2[33.4-35.0]$ & $64.0[62.1-66.0]$ & $35.2[31.1-39.4]$ \\
\hline Opioid analgesics & $54.9[54.2-55.6]$ & 41.0 [39.2-42.9] & 48.9 [46.7-51.1] & 39.2 [33.1-45.3] \\
\hline Other narcotics & $40.6[39.9-41.3]$ & 38.2 [35.9-40.5] & 20.2 [18.6-21.8] & 34.4 [23.8-45.0] \\
\hline Sedatives & 30.2 [29.6-30.9] & $60.7[57.0-64.4]$ & 28.8 [26.7-30.8] & 60.5 [49.1-71.8] \\
\hline Psychotropics & $25.9[25.2-26.5]$ & $44.8[41.1-48.4]$ & 27.5 [25.6-29.5] & $44.0[33.8-54.3]$ \\
\hline Other specified & $9.6[9.2-10.1]$ & $48.6[41.8-55.5]$ & $22.5[20.8-24.2]$ & 28.9 [19.1-38.8] \\
\hline Unspecified & $34.1[33.4-34.7]$ & $-31.9[-30.5--33.2]$ & $34.5[32.5-36.5]$ & $-26.7[-22.5--30.9]$ \\
\hline$>1$ Major drug class ${ }^{d}$ & $43.6[42.9-44.4]$ & $58.8[56.1-61.5]$ & $36.4[34.2-38.5]$ & 60.8 [51.3-70.3] \\
\hline
\end{tabular}

Data from the Multiple Cause of Death files

${ }^{b}$ Adjusted proportions are average predicted values from probit models, where at least one specific drug is assumed to be mentioned for all poisoning deaths (SPECIFY = 1). Models also control for: sex, race (black, other), Hispanic, currently married, education (high school dropout, high school graduate, some college, college graduate), age $(\leq 20,21-30,31-40,41-50,51-60,61-70,71-80,>80)$, day of the week of death, and census region

$c_{\%}$ Difference shows the percentage difference between adjusted proportions and those obtained directly from mentions on death certificates

${ }^{\mathrm{d}}$ Two or more of the drug types: opioid analgesics, other narcotics, sedatives, psychotropics, or other specified drugs

Table 6 Adjusted number of drug poisoning deaths and change vs. reported numbers ${ }^{2}$

\begin{tabular}{|c|c|c|c|c|}
\hline \multirow[t]{3}{*}{ Drug category } & \multicolumn{4}{|l|}{$\#[95 \% \mathrm{Cl}]$} \\
\hline & \multicolumn{2}{|l|}{1999} & \multicolumn{2}{|l|}{2012} \\
\hline & Deaths $^{b}$ & $\Delta$ vs. reported ${ }^{c}$ & Deaths $^{b}$ & $\Delta$ vs. reported ${ }^{c}$ \\
\hline Narcotics & $13,114[12,962-13,265]$ & 3192 [3040-3343] & $33,830[33,560-34,100]$ & 8643 [8373-8913] \\
\hline Opioid analgesics & $5275[5087-5463]$ & 1245 [1057-1433] & $22,534[22,254-22,814]$ & 6527 [6247-6807] \\
\hline Other narcotics & 9820 [9641-9999] & 2683 [2504-2862] & $15,933[15,671-16,196]$ & 4366 [4104-4629] \\
\hline Sedatives & 2633 [2473-2792] & 971 [811-1130] & $12,457[12,185-12,729]$ & 4703 [4431-4975] \\
\hline Psychotropics & $3568[3395-3741]$ & 1102 [929-1275] & $10,798[10,544-11,053]$ & 3331 [3077-3586] \\
\hline Other specified & 1482 [1368-1596] & 311 [197-425] & 4528 [4348-4709] & 1372 [1192-1553] \\
\hline Unspecified & $5930[5786-6154]$ & $-2507[-2691--2323]$ & $14,135[13,874-14,396]$ & $-6477[-6738--6216]$ \\
\hline$>1$ Major drug class $^{\mathrm{d}}$ & 4820 [4627-5013] & 1780 [1587-1973] & $17,670[17,469-18,050]$ & 6584 [6293-6874] \\
\hline
\end{tabular}

${ }^{\mathrm{a}}$ Data from the Multiple Cause of Death files

${ }^{\mathrm{b}}$ Number of drug poisoning deaths involving the specified drug, calculated as the product of the number of drug poisoning deaths in the year multiplied by the adjusted proportion obtained from probit models, where at least one specific drug is assumed to be mentioned for all poisoning deaths (SPECIFY = 1). Models also control for: sex, race (black, other), Hispanic, currently married, education (high school dropout, high school graduate, some college, college graduate), age $(\leq 20,21-30,31-40,41-50,51-60,61-70,71-80,>80)$, day of the week of death, and census region. The calculations use more significant digits for adjusted proportions than are shown on Table 3 , so that there may be small differences from those that would be obtained using rounded estimates ${ }^{\mathrm{C}}$ Difference between adjusted number of deaths and unadjusted number based on death certificate reports

${ }^{\mathrm{d}}$ Two or more of the drug types: opioid analgesics, other narcotics, sedatives, psychotropics, or other specified drugs. 
The adjustments raise the number of deaths involving all specific drug categories, while reducing the role of unspecified drugs. Larger increases were observed in 2012 than in 1999, as expected given the dramatic growth in total drug poisoning mortality, and these differences show the extent to which death certificates inaccurately indicate trends in drug involvement. The biggest absolute disparities were for opioid analgesics: 6527 deaths (95\% CI 6247-6807) in 2012 versus 1245 (95\% CI 1057-1433) in 1999 and combination drug use 6584 deaths (95 \% CI 6293-6874) versus 1780 (95\% CI 1587-1973). However, the greatest relative differences were for sedatives and psychotropic medications. (Additional file 6 shows results for drug subcategories).

\section{Discussion}

The information currently provided on death certificates is inadequate for understanding the nature of the drug poisoning epidemic. Particularly problematic is the frequency with which no specific drug is identified [20]. Since this is more common when death certificates are completed by coroners rather than medical examiners and in states without centralized oversight by a Chief Medical Examiner [21], additional training and standardization in states with low drug specification rates may be helpful. Others have also recommended including more detail on the drugs involved in poisoning deaths, toxicology levels of opioids, whether or not these were prescribed to decedent, and more detailed opioid toxicology ICD categories [22, 23].

Until better information becomes available, the predictive adjustment methods developed here can provide more accurate estimates of specific drug involvement. The adjustments are substantial, as death certificates understated mentions of major classes of drugs by $27 \%$ to $58 \%$ in 1999 and by $34 \%$ to $61 \%$ in 2012 and combination drug use by $59 \%$ in both years, with even larger changes for some subcategories of drugs (e.g. antipsychotics and benzodiazepines). Underlying causes of death are also inaccurately recorded because they are classified based upon reported drug mentions. For example, narcotics was classified as the UCD in $46.4 \%$ of drug poisoning cases in 1999 and $37.6 \%$ in 2012, compared to adjusted rates of $59.6 \%$ and $48 \%$ respectively, using the procedures to described above (Additional file 7).

The adjustment procedures work well, but not perfectly. As evidence of this, reported proportions of exclusive unspecified drug mentions were $21.9 \%$ in 1999 and $24.2 \%$ in 2012, while the adjusted shares were $4.3 \%$ and $3.7 \%$. Completely successful adjustment would reduce the adjusted proportion to zero, indicating that $80 \%$ and $85 \%$ (rather than $100 \%$ ) of such mentions were eliminated. Adjusted proportions calculated under the assumption that drug types were never specified on the death certificates (by predicting probabilities after setting SPECIFY=0) were $96.4 \%$ in 1999 and $96.5 \%$ in 2012, which is close to the $100 \%$ adjusted share occurring with perfect prediction. These favorable results occur even though goodness-of-fit, as measured by the pseudo$\mathrm{R}^{2}$, which ranges between 0.06 and 0.33 , implies a limited ability to predict drug use in individual cases. This does not necessarily indicate inaccuracy in the overall estimates of adjusted proportions, but could be problematic if unobserved determinants of drug involvement were spuriously correlated with county-level specification rates.

Better understanding is also needed of the situations under which the unspecified drug category (T50.9) is reported on death certificates. The procedures developed here adjust for cases where unspecified drugs are exclusively mentioned (i.e., when death certificates report a drug poisoning without identifying any of the drugs involved). However, in many cases there are mentions of both specified and unspecified drugs. These sometimes occur when "multidrug toxicity" or something similar, is listed on one part of the death certificate but with more specific information, such as heroin use, reported on another part (source: personal communication with Robert Anderson, Chief of the Mortality Statistics Branch of the National Center for Health Statistics, October 2, and October 6, 2015).

In 1999, 16,849 US residents died of drug poisoning. By 2012, the number had risen to 41,502. Mentions of opioid analgesic almost quadrupled, from 4030 to 16,007, but the adjusted estimates indicate an even larger increase in opioid involvement from 5275 deaths (95 \% CI 50875463 ) in 1999 to 22,534 (95 \% CI 22,254-22,814) in 2012. These figures imply that these drugs were mentioned in $54.3 \%$ ( $95 \%$ CI, $53.6 \%-55 \%$ ) of fatal drug poisonings in the later year. Such results justify efforts to reduce the negative consequences of prescription opioid use, including establishing prescription drug monitoring programs; restrictions on the dispensing of oxycodone and other controlled substances from pain clinics and online pharmacies; and development of abuse-deterrent drug formulations [24-28]. These endeavors have been somewhat successful. Drug poisoning deaths in Florida decreased $17 \%$ between 2010 and 2013, with a $52 \%$ decline in fatalities involving oxycodone following aggressive efforts to reverse the proliferation of pain clinics, prohibit the dispensing of schedule II or III drugs from physician offices, and other measures [29]. Deaths involving methadone peaked in 2007 and then declined along with a fall in the amount of methadone distributed nationally [5]. However, the accomplishments are incomplete. After Florida's crackdown, some pain clinic owners moved out of the state or found ways to circumvent the laws, and it is questionable whether prescription drug monitoring programs have reduced drug poisoning deaths $[24,29,30]$. Most 
notably, fatalities involving heroin have doubled since 2004, raising the possibility of the substitution of heroin for prescription opioids, although evidence on this is conflicting [7, 31, 32].

Nor should attention be limited to the risks of prescription opioids. Deaths involving sedatives, especially benzodiazepines, have grown even faster in percentage terms, and involvement of these drugs and of psychotropic medications is particularly severely understated on death certificates. Attention has been paid to the role of non-opioids and to combination drug use, but a better understanding is needed since opioids and benzodiazepines are often combined, with greater resulting health risks than the use of either alone [2, 8, 33, 34]. In a more general sense, comprehensive efforts to reduce drug fatalities should account for potential therapeutic benefits and substitution between drugs, the frequency of combination drug use, and the heterogeneity in levels and growth of drug mortality across geographic areas and demographic groups $[2,35,36]$.

The results of this analysis are subject to additional caveats. First, the drugs identified as contributing to fatal drug poisonings may sometimes be reported inaccurately. For instance, heroin use may sometimes be misattributed to morphine or codeine, because heroin metabolizes into morphine and codeine may be detected as an impurity in either morphine or heroin [37]. Second, some deaths could be misclassified as being due to nondrug causes, and therefore be excluded from the analysis, while others that are defined as drug poisonings may actually primarily result from non-drug causes. Third, the adjustment procedures do not fully correct for cases where death certificates mention both unspecified and specified types of drugs. Fourth, information on specific drug involvement was not available on death certificates, and so not examined here, for the small number of cases (732 in 1999 and 637 in 2012) where the underlying cause was classified as a mental or behavior disorder due to drug use (ICD-10 codes F11 through F16). Fifth, while population-wide adjusted proportions are provided, it would be useful to obtain estimates for subcategories. However, the precision of such estimates will be limited for groups where the number of deaths is small, and this is likely to make it difficult to construct age-adjusted proportions using finely defined age categories. Finally, future research should examine whether these adjustments change the estimated consequences of policies potentially affecting drug deaths, such as those related to prescription drug monitoring programs, internet pharmacies, and medical cannabis laws [29, 38, 39].

\section{Conclusions}

Death certificates substantially understate the frequency of involvement of opioid analgesics, sedatives, psychotropics, and combinations of drugs in fatal drug poisonings. Adjustment procedures that account for cases where only unspecified drugs are reported on death certificates provide more accurate and informative estimates.

\section{Additional files} Additional file 1: Percent of drug poisoning deaths with at least
one drug specified ${ }^{\mathbf{a}}$. (DOCX $\left.39 \mathrm{~kb}\right)$

Additional file 2: Actual and predicted drug mentions ${ }^{\mathrm{a}}$. (DOCX $35 \mathrm{~kb}$ ) Additional file 3: Adjusted drug involvement, with and without controls for manner of death ${ }^{\mathrm{a}}$. (DOCX $39 \mathrm{~kb}$ )

Additional file 4: Reported drug involvement by manner of death ${ }^{\mathrm{a}}$. (DOCX $36 \mathrm{~kb}$ )

Additional file 5: Reported drug involvement by age ${ }^{a}$. (DOCX $37 \mathrm{~kb}$ ) Additional file 6: Adjusted number of drug poisoning deaths and percent change vs. reported numbers for drug subcategories ${ }^{a}$. (DOCX $41 \mathrm{~kb}$ )

Additional file 7: Reported and adjusted underlying cause of death shares $^{\mathrm{a}}$. (DOCX $\left.37 \mathrm{~kb}\right)$

Competing interests

The author declares that he has no competing interests.

Acknowledgements

Financial support for this research was received from the University of Virginia Bankard Fund.

Received: 2 March 2015 Accepted: 12 January 2016

Published online: 15 January 2016

References

1. Warner M, Chen LH, Makuc DM, Anderson RN, Miniño AM. Drug poisoning deaths in the United States, 1980-2008. NCHS data brief, No. 81. Hyattsville, MD: National Center for Health Statistics; 2011.

2. Chen LH, Hedegaard $H$, Warner M. Drug-Poisoning deaths involving opioid analgesics: United States, 1999-2011. NCHS data brief, No. 166. Hyattsville, MD: National Center for Health Statistics. 2014.

3. Case A, Deaton A. Rising morbidity and mortality in midlife among white non-Hispanic Americans in the $21^{\text {st }}$ century. Proc Natl Acad Sci USA. 2015. doi:10.1073/pnas.1518393112.

4. CDC. Vital Signs: Overdoses of prescription opioid pain relievers - United States, 1999-2008. MMWR Morb Mortal Wkly Rep. 2011;60(43):1487-92.

5. CDC. Vital Signs: Risk for overdose from methadone used for pain relief United States, 1999-2010. MMWR Morb Mortal Wkly Rep. 2012;61(26):493-7.

6. Volkow ND, Frieden TR, Hyde PS, Cha SS. Medication-assisted therapies tackling the opioid overdose epidemic. N Engl J Med. 2014;370(22):2063-6.

7. Hardesty C. White House summit on the opioid epidemic. Washington DC: Office of National Drug Policy; 2014. http://www.whitehouse.gov/blog/ 2014/06/19/white-house-summit-opioid-epidemic. Accessed August 27, 2014.

8. Jones CM, Mack KA, Paulozzi L. Pharmaceutical overdose deaths, United States, 2010. JAMA. 2013;309(7):657-9.

9. Paulozzi LJ, Mack KA, Hockenberry JM. Vital Signs: Variation among states in prescribing of opioid pain relievers and benzodiazepines - United States, 2012. MMWR Morb Mortal Wkly Rep. 2014;63(26):563-8.

10. CDC Wonder. Multiple cause of death 1999-2011. http://wonder.cdc.gov. Accessed August 22, 2014.

11. Anderson RN, Miniño AM, Hoyert DL, Rosenberg HM. Comparability of cause of death between ICD-9 and ICD-10: preliminary estimates. Natl Vital Stat Rep. 2001;49(2):1-32.

12. CDC, National Center for Health Statistics. Instructions for completing the cause-of-death section of the death certificate. http://www.cdc.gov/nchs/ data/dvs/blue_form.pdf. Accessed August 22, 2014.

13. World Health Organization. International classification of diseases (ICD). http://www.who.int/classifications/icd/en/. 2014. Accessed August 26, 2014. 
14. ChiroCode Institute. ICD-10-CM (ICD10) Diagnosis codes - T codes. https://www.chirocode.com. 2014. Accessed August 26, 2014.

15. Stock JH, Watson MW. Introduction to econometrics. 3rd ed. Boston, MA: Addison-Wesley; 2011.

16. Rockett IR, Kapusta ND, Coben JH. Beyond suicide: action needed to improve self-injury mortality accounting. JAMA Psychiatry. 2014;71(3):231-2.

17. Rockett IR, Smith GS, Caine ED, Kapusta ND, Hanzlic RL, Larkin GL, et al. Confronting death from self-intoxication (DDSI): prevention through a better definition. AJPH. 2014;104(12):e49-55.

18. McFadden D. Conditional logit analysis of qualitative choice behavior. In: Zarembaka P, editor. Frontiers in Econometrics. New York: Academic Press; 1974. p. 105-42.

19. StataCorp. Stata statistical software: release 14. College Station, TX: StataCorp LP; 2015.

20. Slavova S, O'Brien DB, Creppage K, Dao D, Fondario A, Haile E, et al. Drug overdose deaths: let's get specific. Public Health Rep. 2015;130(4):339-42.

21. Warner M, Paulozzi LJ, Nolte KB, Davis GG, Nelson LS. State variation in certifying manner of death and drugs involved in drug intoxication deaths. Acad Forensic Pathol. 2013;3(2):231-7.

22. Webster $L R$, Dasgupta N. Obtaining adequate data to determine causes of opioid-related overdose deaths. Pain Med. 2011;12 Suppl 2:S86-92.

23. Goldberger BA, Maxwell JC, Campbell A, Wilford BB. Uniform standards and case definitions for classifying opioid-related Deaths: recommendations by a Samhsa consensus panel. J Addict Dis. 2013;32(3):231-43.

24. Finklea KM, Bagalman E, Sacco LN. Prescription drug monitoring programs. Washington, DC: Library of Congress, Congressional Research Service; 2013.

25. CDC. Opioids drive continued increase in drug overdose deaths. 2013 February 20. http://www.cdc.gov/media/releases/2013/p0220_drug_ overdose_deaths.html. Accessed August 27, 2014.

26. Rannazzisi JT. Testimony for "Curbing prescription drug abuse in medicare". Hearing before the Committee on Homeland Security and Governmental Affairs, United States Senate, 113th Congress, June 24, 2013, retrieved from: http://www.gpo.gov/fdsys/pkg/CHRG-113shrg82571/pdf/CHRG113shrg82571.pdf. August 26, 2014.

27. Kirschner N, Ginsburg J, Sulmasy LS, Health and Public Policy Committee of the American College of Physicians. Prescription drug abuse: executive summary of a policy position paper from the American College of Physicians. Ann Intern Med. 2014;160(3):198-200.

28. Johnson H, Paulozzi L, Porucznik C, Mack K, Herter B, Hal Johnson Consulting and Division of Disease Control and Health Promotion, Florida Department of Health. Decline in drug overdose deaths after state policy changes - Florida, 2010-2012. MMWR Morb Mortal Wkly Rep. 2014;63(26):569-74.

29. Paulozzi L, Kilbourne EM, Desai HA. Prescription drug monitoring programs and death rates from drug overdose. Pain Med. 2011;12(5):747-54.

30. Gugelmann HM, Perrone J. Can prescription drug monitoring programs help limit opioid abuse? JAMA. 2011;306(20):2258-9.

31. Cicero TJ, Ellis MS, Surratt HL. Effect of abuse-deterrent formulation of OxyContin. N Engl J Med. 2012;367(2):187-9.

32. Rudd RA, Paulozzi LJ, Bauer MJ, Burleson RW, Carlson RE, Dao D, et al. Increase in heroin overdose deaths - 28 states, 2010 to 2012. MMWR Morb Mortal Wkly Rep. 2014;63(39):849-54.

33. Jones JD, Mogali S, Comer SD. Polydrug abuse: a review of opioid and benzodiazepine combination use. Drug Alcohol Depend. 2012;125(1-2):8-18,

34. Park TW, Saitz R, Ganoczy D, Ilgen M, Bohnert AS. Benzodiazepine prescribing patterns and deaths from drug overdose among US veterans receiving opioid analgesics: case-cohort study. BMJ. 2015;350:h2698.

35. CDC. Vital signs: overdoses of prescription opioid pain relievers and other drugs among women-United States, 1999-2010. MMWR Morb Mortal Wkly Rep. 2013;62(26):537-42.

36. Rossen $L M$, Khan $D$, Warner M. Trends and geographic patterns in drugpoisoning death rates in the U.S., 1999-2009. Am J Prev Med. 2013;45(6):e19-25.

37. Mertz KJ, Janssen JK, Williams KE. Underrepresentation of heroin involvement in unintentional drug overdose deaths in Allegheny County. PA J Forensic Sci. 2014;59(6):1583-5.

38. Jena $A B$, Goldman DP. Growing Internet use may help to explain the rise in prescription drug abuse in the United States. Health Aff. 2011;30(6):1192-9.

39. Bachhuber MA, Saloner B, Cunningham CO, Barry CL. Medical cannabis laws and opioid analgesic overdose mortality in the United States, 1999-2010. JAMA Intern Med. Published online August 25, 2014. doi:10.1001/jamainternmed.2014.4005.

\section{Submit your next manuscript to BioMed Central and we will help you at every step:}

- We accept pre-submission inquiries

- Our selector tool helps you to find the most relevant journal

- We provide round the clock customer support

- Convenient online submission

- Thorough peer review

- Inclusion in PubMed and all major indexing services

- Maximum visibility for your research

Submit your manuscript at www.biomedcentral.com/submit
() BioMed Central 